\title{
La nueva formación de profesionales: sobre la competencia profesional y la competencia del estudiante de enfermería
}

\section{New professional training: professional competence and the nursing student competence}

\author{
Anna Falcó Pegueroles \\ Escola Universitària d'Infermeria Gimbernat (adscrito a la UAB). Sant Cugat del Vallès (Barcelona)
}

La idea de competencia profesional está, desde hace unos años, presente en varias profesiones, entre ellas la enfermería y la medicina. La reciente publicación de trabajos y artículos sobre el tema en la literatura científica en lengua castellana demuestra la creciente implicación de los profesionales en la necesidad de aplicar, tanto desde la perspectiva asistencial como la educativa, fórmulas para crear profesionales competentes.

La transformación impulsada por las directrices europeas de enseñanza superior que está experimentando el mundo universitario, ha supuesto, entre otras, la organización de las enseñanzas en función del aprendizaje y la introducción de la educación basada en las competencias del alumno. La definición de las competencias del estudiante es una tarea fundamental, que permite coordinar la formación y determinar la priorización de los objetivos de aprendizaje, tanto en pregrado como en postgrado. Es importante constatar el esfuerzo de diversas escuelas para introducir cambios en los planes de estudio y nuevas estrategias metodológicas encaminadas al objetivo de una educación basada en las competencias finales del alumno. Algunos ejemplos son los nuevos planteamientos en el diseño de programas y la introducción de estrategias como el aprendizaje basado en problemas, los practicum o los sistemas de evaluación basado en las competencias.
The idea of professional competence has recently emerged in several fields, among them nursing and medicine.

The publication of studies and articles on the subject in the scientific literature in Spanish shows the increasing involvement of professionals in the implementation of formulae in the areas of both care and education in order to train competent professionals.

With the transformations in the university world, and the introduction of the higher education guidelines in Europe there has been a shift in emphasis in the organisation of teaching towards the learning process with the introduction of competence-based education. The definition of students' competence is a fundamental task which allows specialists in nursing education to coordinate training and to determine the establishment of priorities for learning in both preand post-graduate studies.

Several colleges have gone to great lengths to introduce changes in their curriculum and new methodological strategies promoting competencebased education. Examples are the new program designs and the introduction of strategies such as problem-based learning, and competence-based practicums and assessment systems.

Key words: Nursing, Student competence, Competence-based education, outcome-oriented-education, University teaching

\section{INTRODUCCIÓN}

Correspondencia:

Anna Falcó Pegueroles

EUI Gimbernat

Vial Interpolar del Vallès, $\mathrm{s} / \mathrm{n}$.

08190 Sant Cugat del Vallès. (Barcelona)

e-mail: anna.falco@cesc.es
La idea de competencia y de profesional competente está, desde hace unos años, presente en varias profesiones, entre ellas la enfermería y la medicina. Se habla de competencia clínica, de competencia profesional, de competencias del estudiante, de edu- 
cación basada en las competencias finales del alumno, de la evaluación de la competencia, de los instrumentos y sistemas de acreditación de la competencia, de la gestión y la retribución de la competencia profesional.

La revisión de la literatura científica inglesa de la última década, pone de manifiesto la necesidad de definir estos términos y clarificar conceptos. La reciente publicación de trabajos y artículos sobre el tema en la literatura científica en lengua castellana demuestra la creciente implicación de los profesionales en la necesidad de aplicar, tanto desde la perspectiva asistencial como la educativa, fórmulas para crear profesionales competentes. Es, por ello, un debate que está muy presente en el mundo sanitario actual, en los ámbitos de la asistencia, la gestión y la docencia.

La definición del concepto de competencia es compleja. Como afirma Kane ${ }^{1}$, un profesional es competente en la medida en que utiliza los conocimientos, las habilidades, las actitudes y el buen juicio asociados a su profesión, con la finalidad de poder desarrollarla de forma eficaz en aquellas situaciones que corresponden al campo de su práctica. La competencia, pues, pertenece al área del saber hacer ${ }^{2}$, a la demostración en sí misma, no tanto a la acreditación o a la intención.

Diversos autores en enfermería ${ }^{3-5}$ han reiterado la necesidad de clarificar diferentes términos referentes a la idea de competencia, como son la competencia (competence en inglés), la capacidad (competency) y la ejecución o desempeño (performance). Es decir, un profesional puede acreditar el ser competente, pero ante una situación real no demostrarlo: no demostrar el saber, el saber estar, el saber hacer y el buen juicio enfermero, por lo que no responde al nivel profesional de calidad exigible en aquella situación. Newble ${ }^{6}$ explicó la competencia clínica diferenciando entre lo que el estudiante o el médico debería ser capaz de hacer en un nivel esperado de logro y el desempeño clínico, lo que el estudiante o el médico hace en la práctica real.

\section{Competencias del estudiante de enfermería}

En los últimos años, la reforma universitaria impulsada por las nuevas directrices europeas de enseñanza superior está organizando la formación en función de las competencias finales del alumno, modificando la consideración de la enseñanza exclusivamente fundamentada en la tarea docente a la basada en el aprendizaje y trabajo del alumno. Los anglosajones han definido este tipo de educación como outcome-oriented-education, la educación basada en el producto o en los resultados. Como afirma Palés ${ }^{7}$, este tipo de educación no es una educación centrada en sí misma, sino que está determinada por un referente externo: las necesidades de la sociedad.

Las ventajas de la educación basada en las competencias finales del estudiante de enfermería, y medicina, entre otras disciplinas, son numerosas: potencia la responsabilidad del alumno haciéndolo partícipe de manera activa en su proceso de aprendizaje; permite la aplicación de metodologías didácticas distintas en función de la materia y la dinámica del grupo; obliga a diseñar de manera práctica y coherente las materias, permitiendo la distinción entre lo esencial y lo no esencial, racionalizando los recursos y proporcionando mayor cohesión en el currículum formativo; también potencia el papel del profesor como agente especialista en el diagnóstico y prescripción del aprendizaje, agente facilitador de recursos, y le permite ser consciente de cuál es su contribución al conjunto del currículum y su responsabilidad en el proceso de aprendizaje.

Las competencias del alumno determinan aquello que deben aprender los estudiantes y aquello que debe ser evaluado, además de constituir el eje de todo el proceso de enseñanza-aprendizaje. Su definición es una tarea fundamental, tanto para los agentes implicados en la formación del futuro profesional como para las instituciones sanitarias y la sociedad en general. Como afirma Nolla ${ }^{8}$, esta definición debe presidir la construcción y el desarrollo curricular, dando luz a contenidos, metodología docente y estrategias educativas, estrechando las relaciones entre teoría y praxis asistencial y profesional en el ámbito de la salud.

La definición de las competencias del estudiante de enfermería debe estar orientada por las competencias profesionales y por la consideración de varios elementos como son: los factores extraprofesionales, que definen el ideal de profesional competente para el alumno; y la progresión de éste en el aprendizaje a lo largo de la Diplomatura. Lo que se enseña al estudiante sobre la profesión, lo que ve y hace en los centros de prácticas, y la propia experimentación-socialización a lo largo de la carrera universitaria, son algunos de los parámetros que incorpora el estudiante para configurar su ideal de competencia profesional. El aprendizaje del alumno, en tanto que experiencia individual, es la que verdaderamente definirá el concepto y el desempeño de la enfermería en éste. 
Por otra parte, también debe considerarse la perspectiva temporal en la definición y evaluación de las competencias del estudiante. Como afirma Ramritu ${ }^{4}$ hay una complejidad creciente en las percepciones e interpretaciones de la competencia clínica de las enfermeras: desde la apreciación de ésta como una práctica segura, por parte de los recién titulados, hasta la consideración cómo conocimiento, independencia, habilidad, gestión de recursos y tiempo, para las enfermeras más experimentadas. Esta complejidad supone un factor imprescindible para orientar y coordinar la formación, además de determinar la priorización de los objetivos de aprendizaje, tanto en la formación de pregrado como en la de postgrado.

\section{Estado actual sobre la competencia profesional y la educación basada en las competencias del alumno en las Escuelas de Enfermería españolas}

Algunas instituciones sanitarias estatales y autonómicas están promocionando, desde hace unos años, la formación de grupos de trabajo para elaborar documentos marco de las profesiones, que incluyen la definición de las competencias profesionales en enfermería. Un ejemplo es el documento "Competències de la professió d'infermeria", desarrollado por el Consell Català d'Especialitats y publicado por el Institut d'Estudis de la Salut, en el ámbito de Cataluña. Este documento, de referencia obligada para la enfermería española, especialmen- te la catalana, concreta las competencias de la enfermería siendo un primer marco orientador.

En el ámbito de las Escuelas Universitarias de Enfermería no hay constancia aún de un documento marco que defina las competencias de los estudiantes de enfermería a nivel estatal, aunque hay algunas publicaciones que pueden orientar trabajos en esta línea ${ }^{5,10,11}$. Como afirma $\mathrm{Manso}^{12}$, en la práctica docente las innovaciones en la formación de profesionales sanitarios se centran más en los aspectos formales y metodológicos, como los créditos, los horarios, los contenidos, que en la definición concreta de lo que deben aprender los alumnos y la evaluación real de las competencias adquiridas que se certificaran ante la sociedad. A pesar de no haber constancia de un documento de estas características ni en Enfermería ni en otras carreras como Medicina o Fisioterapia, si que es destacable el esfuerzo de diversas escuelas para introducir cambios en los planes de estudio y nuevas estrategias metodológicas encaminadas a educar a partir de las competencias finales del alumno, siguiendo las directrices europeas de enseñanza superior.

El establecer las competencias del alumno ya sea de manera detallada o globalmente, ha modificado sustancialmente la manera de planificar la enseñanza por parte del docente (Figura I). Así, las necesidades sociales y profesionales son las que deben orientar la definición de las competencias del estudiante a través de los objetivos de aprendizaje, y éstos inspirar el diseño de los programas, la defi-

Figura I. Diseños tradicional y actual del proceso de enseñanza-aprendizaje para algunas asignaturas que configuran el currículum de enfermería

Diseño tradicional
$\begin{gathered}\text { Referencias bibliográficas, } \\ \text { otros programas, experiencia } \\ \text { propia el docente, necesidades profesionales }\end{gathered}$


nición de los objetivos docentes y la determinación de los criterios de evaluación. Es este un planteamiento distinto al tradicional, que permite optimizar los contenidos y el tiempo que se dispone para enseñarlos, además de constituir la verdadera introducción y aplicación de la educación basada en las competencias del estudiante.

Otros ejemplos de estas nuevas estrategias metodológicas son la introducción del Aprendizaje Basado en Problemas, los Practicums, el diario reflexivo $^{13}$ o la consideración de Sistemas de Evaluación basado en las competencias ${ }^{14}$.

La educación basada en las competencias del estudiante está generando la necesaria e inevitable renovación de los currículums formativos en ciencias de la salud, y una nueva organización de las tareas del alumno y del profesor. En la Diplomatura de Enfermería, esta renovación se suma a las modificaciones del plan de estudios de los últimos años, con el objetivo de equipar la carga lectiva a la del resto de Europa.

La introducción de nuevos diseños y estrategias metodológicas encaminadas a vertebrar las asignaturas hacia las competencias finales del estudiante de enfermería, hace cada vez más necesaria su exhaustiva definición y su concreción operativa en los objetivos de aprendizaje. Estos objetivos establecen las características de la acción del discente, e instituyen el nivel de competencia exigible en el programa diseñado. Se añade así a la tarea docente la búsqueda, la selección y el análisis de los objetivos por cada temario, asignatura y materia, de manera longitudinal y transversal, siendo imprescindible destinar más tiempo para poder organizar los contenidos y las materias a partir de esta nueva configuración. Por todo ello, será necesario el trabajo en equipo de los profesionales encargados de la formación a nivel autonómico y estatal, además de destinar, por parte de las escuelas de enfermería, más recursos para poder hacer frente al reto de formar a las enfermeras competentes que la sociedad demanda.

\section{BIBLIOGRAFÍA}

1. Kane MT. The assessment of professional competence. Education and the Health Professions 1992;15:163-182.

2. Elías A, Esteve C, Esteve N, Pagés J et al. Competencias de la profesión enfermera. Educación Médica 1999;2 (2): 89-94.

3 . While A. Competence versus Performance: which is more important?. Journal of Advanced Nursing 1994;20:525-531.

4. Ramritu PL, Barnard A. Cómo comprenden la competencia los enfermeros recién licenciados. Internacional Nursing Review (versión española) 2001;48(1):51-61.

5. Watson R, Stimpson A, Toping A, Porock D. Clinical competence assessment in nursing: a systematic review of the literature. Journal of Advanced Nursing 2002;39(5):421-431.

6. Newble D. Assessing clinical competence ar the undergraduate level. Medical Education 1992;26:504-511.

7. Palés J. La educación médica basada en las competencias finales del estudiante. Educación Médica 2001;4(1):1-2.

8. Nolla M, Palés J, Gual A. Desarrollo de las competencias profesionales. Educación Médica 2002;5(2):76-81.

9. Consell Català d'Especialitats en Ciències de la Salut. Competències de la professió d'Infermeria. Barcelona: Autor, 1997.

10. Vázquez J. Educación basada en competencias clínicas. La experiencia de la Universidad de Monterrey. Educación Médica 2001;4(3):100-103.

11. Palés J, Vallés A, Cardellanch F, Gomar C, Estrach MT et al. Habilidades y procedimientos básicos a adquirir por los estudiantes de la Facultad de Medicina de la Universidad de Barcelona. Educación Médica 2001;4(2):72-81.

12. Manso JM. ¿Qué enseñar en ciencias de la salud? Técnicas para definir competencias y perfiles profesionales (I). Educación Médica 2000;3(2):61-68.

13. Betolaza E, Alonso I. El diario reflexivo y el autoaprendizaje tutorizado. Metas 2002;45(2):14-18.

14. Pulpón AM, Solá M, Gispert R, Martínez JM. Evaluación de la competencia clínica en enfermería mediante pacientes simulados / estandarizados. Enfermería Clínica 1997; 7 (4):157-162. 